\title{
Influence of Pressure and Temperature on the Velocity of a Turbulent Jet Flow 3D
}

Flávia Aparecida Reitz Cardoso ${ }^{1 *}$, Regiani Aparecida de Almeida ${ }^{2}$, Ricardo Vicente de Paula Rezende ${ }^{3}$, Lúcio Cardozo-Filho ${ }^{2}$, Dirceu Noriler ${ }^{4}$, Henry França Meier ${ }^{4}$ and Vladimir Ferreira Cabral ${ }^{2}$

1. Department of Mathematics, Federal Technological University of Paraná, Campo Mourao 87301-005, Brazil

2. Department of Chemical Engineering, State University of Maringa, Maringa 87020-900, Brazil

3. Department of Chemical Engineering, Federal University of Santa Catarina, Florianopolis 88040-970, Brazil

4. Department of Chemical Engineering, Regional University of Blumenau, Blumenau 89030-000, Brazil

Received: March 17, 2014 / Accepted: April 06, 2014 / Published: April 25, 2014.

\begin{abstract}
To investigate the influence of pressure and temperature on the jet velocity of a three-dimensional flow was the main goal of this study. Using a precipitation chamber with approximate capacity of $600 \mathrm{~mL}$, it was studied the thermodynamic behavior of supercritical carbon dioxide mixture, dichloromethane and grape seed extract via SAS (supercritical antisolvent process). For the numerical solution, the Navier-Stokes equations were used along with the model of turbulence $k$ - $\varepsilon$ and Peng-Robinson equation of state with quadratic mixing rules of Van der Waals. The method of Chung was employed to determine the viscosity, thermal conductivity and mass diffusivity of the flow numerically solved through commercial code based on CFD (computational fluid dynamics). Simulations for pressures between $80 \mathrm{bar}$ and $160 \mathrm{bar}$ and temperatures between $308.15 \mathrm{~K}$ and $318.15 \mathrm{~K}$ showed large variations in the jet velocity, an important property in the dynamic mixing process that involves the size, size distribution and particle morphology.
\end{abstract}

Key words: Supercritical fluid, CFD, mathematical modeling.

\section{Nomenclature}

$\begin{array}{ll}k & \text { Turbulent kinetic energy }\left(\mathrm{m}^{2} \cdot \mathrm{s}^{-2}\right) \\ p & \text { Pressure (bar) } \\ R & \text { Universal gas constant }\left(\mathrm{J} \cdot \mathrm{mol}^{-1} \cdot \mathrm{K}^{-1}\right) \\ R & \text { Radial direction }(\mathrm{m}) \\ T & \text { Temperature }(\mathrm{K}) \\ t & \text { Time }(\mathrm{s}) \\ v & \text { Velocity }\left(\mathrm{m} \cdot \mathrm{s}^{-1}\right) \\ x & \text { Axial direction }(\mathrm{m}) \\ \rho & \text { Density }\left(\mathrm{kg} \cdot \mathrm{m}^{-3}\right) \\ \sigma & \text { Prandtl number }(-) \\ \omega & \text { Fugacity }(-) \\ \lambda & \text { Thermal conductivity }\left(\mathrm{W} \cdot \mathrm{m}^{-1} \cdot \mathrm{K}^{-1}\right) \\ \tau & \text { Tensor stress }(\mathrm{pa}) \\ \varepsilon & \text { Rate of turbulent } \mathrm{kinetic} \mathrm{energy} \mathrm{dissipation}\left(\mathrm{m}^{2} \cdot \mathrm{s}^{-3}\right) \\ v & \text { Molar volume }\left(\mathrm{m}^{3} \cdot \mathrm{mol}{ }^{-1}\right)\end{array}$

*Corresponding author: Flávia Aparecida Reitz Cardoso, Ph.D. Student, research fields: computational fluid dynamics and supercritical technology. E-mail: flaviareitz@gmail.com.

\section{Introduction}

Among the existing methods of preparing nanoparticles, SAS (supercritical antisolvent precipitation) is becoming increasingly important due to the large amount of chemicals that can be processed with this technology [1-3]. Above the critical point of the mixture, there condition of complete miscibility, typically in the range of $8-16 \mathrm{MPa}$, where $\mathrm{CO}_{2}$ is completely miscible with many organic solvents and supercritical turbulent mixing is the most important issue. The SAS process involves mixing $\mathrm{CO}_{2}$ and organic solution (solute dissolved in an organic solvent) from two outputs of a coaxial nozzle system, to obtain the desired conditions to produce thermodynamic supersaturation and particle formation, and the resulting PSD (particle size distribution) is affected by blending [4]. While blending effects are well known 
to those based on precipitation in liquids [5], such effects are not very often reported in supercritical systems.

Despite a number of studies that support this evidence under the experimental point of view, little research is available in the context of numerical modeling. A mathematical model for mass transfer between a droplet of organic solvent and a compressed antisolvent was developed by Werling and Debenedetti [6], while Chavez et al. [7] have proposed a methodology to calculate the time scales involved. A more elaborate model, solved by CFD (computational fluid dynamics) was proposed by Martin and Cocero [3], in which a turbulence model was solved in conjunction with mass balances and took into account the effect of mass transfer in the system. The authors employed a locking to solve the PBE (population balance equations) [8]. The results were strongly affected by the value of solid-liquid surface tension. Forecasts obtained from models are valuable for understanding the process behavior at a large-scale industrial level. Henczka et al. [9] studied numerically supercritical antisolvent precipitation of paracetamol with carbon dioxide as antisolvent and took into account the heat of mixing in the studied system. The PSD was calculated using the standard method of moments, and the interaction between mixing and precipitation was taken into account with the $\beta$-PDF approach along with the turbulent model of Baldyga mixer [10]. This first attempt showed the importance of including mixing effects in the model, and highlighted some limitations of the approaches available. Since the micromixing models available [1-8], needed to explain the interactions between turbulence and particle formation are valid only for standard fluids, and its extension to supercritical fluids is not simple [11]. It is known that the fluid properties change drastically near and above the critical point, then the dynamic behavior of the fluid also changes and this directly influences the mixing at all scales.
A large number of publications on the micronization of different materials with the SAS process appears concerned with the experimental analysis of the effects of operating conditions on the size distribution and morphology of the particles, and how this type of analysis is often difficult, because the interactions between these parameters. This study aims to provide a mathematical formulation capable of describing the influence of pressure and temperature on the jet velocity of a three-dimensional flow phenomena that influence the turbulent mixing observed in the SAS process. Once validated, the effects of thermodynamic properties can be used for modeling, scale-up and optimization of systems, with acceptable precision for engineering calculations.

\section{Governing Equations}

\subsection{Thermodynamics}

The density of the mixture is described by Peng-Robinson equation of state with the quadratic mixing rules of Van der Waals:

$$
p=\frac{R T}{v-b_{m}}=\frac{a_{m} T}{v\left(v+b_{m}\right)+b_{m}\left(v-b_{m}\right)}
$$

where, $p$ the pressure, $v$ molar volume, $T$ temperature, $a_{m}$ and $b_{m}$ the constants of the equation for the case of mixtures. These constants are obtained by mixing rule is given by $a_{m}=a(T)=\sum_{\mathrm{i}} \sum_{\mathrm{j}} x_{i} x_{j} a_{i j}$ and $b_{m}=$ $b(T)=\sum_{\mathrm{i}} \sum_{\mathrm{j}} x_{i} x_{j} a_{i j}$, where, $x_{i}$ is the molar concentration of the component, $i$ is combination rulesfraction: $\quad a_{i j}=\sqrt{a_{i} a_{j}}\left(1-k_{i j}\right)$ and $b_{i j}=$ $\frac{b_{i}+b_{j}}{2}\left(1-l_{i j}\right)$.

This formulation allows to study the importance of solving the resulting system of equations in non-isothermal regime.

\subsection{Hydrodynamics}

The conservation equations are expressed here to a differential control volume fixed in three dimensional space of the flow of a mixture in the supercritical state and considering the turbulent flow, a variable can be 
considered as the sum of an average value plus a fluctuation $\phi=\tilde{\phi}+\phi^{\text {创 }}=\bar{\phi}+\phi$. The equation of continuity in Cartesian coordinates for a system with variable density in steady state is given by:

$$
\partial i\left(\bar{\rho} \tilde{u}_{i}\right)=0
$$

where, $\tilde{u}_{i}$, with $i \in\{1,2,3\}$, represents the velocity vector mean component $\tilde{u}$ to the position vector components respectively.

The equation of motion that takes into account the density and viscosity are variable.

$$
\partial i\left(\bar{\rho} \tilde{u}_{i} \tilde{u}_{j}\right)=\partial j \bar{P}+\partial j\left(\tilde{\tau}_{i j}-\bar{\rho} u_{i}^{\prime} u_{j}^{\prime}\right)
$$

where, $-\bar{\rho} u_{i} u_{j}^{\prime}$ is the Reynolds tensor, resolved by the turbulence model and the two equations

$$
\tilde{\tau}_{i j}=\mu\left\{2\left(\frac{\partial \tilde{u_{i}}}{\partial x_{j}}+\frac{\partial \tilde{u}_{j}}{\partial x_{i}}\right)-\frac{2}{3} \delta_{i j} \frac{\partial \tilde{u_{k}}}{\partial x_{k}}\right\}
$$

Considering the viscous dissipation of energy Eq. (4) and species conservation Eq. (5) are given by:

$$
\frac{\partial}{\partial x_{i}}(\bar{\rho} \tilde{u} \tilde{h})=\frac{\partial}{\partial x_{i}}\left(\frac{k_{M}+k_{T}}{C_{p}}+\bar{\rho} u_{j} \cdot \tilde{h}\right)+\tilde{\tau_{i j}} \frac{\partial}{\partial x_{j}} \tilde{u_{i}}
$$

and

$$
\frac{\partial}{\partial x_{j}}\left(\bar{\rho} \tilde{u}_{j} \tilde{y}_{i}+\bar{\rho} u_{j} \text { 创 }_{i}^{\prime}\right)=\frac{\partial}{\partial x_{j}}\left(\bar{\rho} D_{m} \frac{\partial y_{i}^{\prime}}{\partial x_{j}}\right)
$$

where, $\tilde{h}$ is the enthalpy, the mass fraction $y_{i}$ of component $i$ and $D_{m}$ the molecular diffusion coefficient.

\subsection{Model of Turbulence}

The model of turbulence $k-\varepsilon$ for two equations was used in this study, and consists of two semiempirical Eqs. (7) and (8) for the turbulent kinetic energy $k$ and for dissipation rate of turbulent kinetic energy $\varepsilon$ :

$$
\underbrace{\frac{\partial}{\partial x_{j}}\left(\rho k \tilde{u}_{j}\right)}_{I}=\underbrace{\frac{\partial}{\partial x_{j}}\left[\left(\mu+\frac{\mu_{T}}{\sigma_{k}}\right) \frac{\partial}{\partial x_{j}} k\right]}_{I I}+\underbrace{P_{k}}_{I I I}-\underbrace{\rho \varepsilon}_{I V}
$$

$$
\underbrace{\frac{\partial}{\partial x_{j}}\left(\rho \varepsilon \tilde{u}_{j}\right)}_{I}=\underbrace{\frac{\partial}{\partial x_{j}}\left[\left(\mu+\frac{\mu_{T}}{\sigma_{k}}\right) \frac{\partial}{\partial x_{j}} \varepsilon\right]}_{I I}+\underbrace{C_{\varepsilon 1} \frac{\varepsilon}{k} P_{k}}_{I I I}-\underbrace{C_{\varepsilon 2} \frac{\rho \varepsilon^{2}}{k}}_{I V},
$$

where, $\varepsilon$ is the dissipation per unit mass. In Eqs. (7) and (8), the term $I$ is the convective transport of $k$ or $\varepsilon$; the term II corresponds to diffusive transport; the term $I I I$ is the production rate; and the term $I V$ is the destruction rate. $P_{k}$ is a term of turbulence production due to viscous forces.

$$
P_{k}=\mu_{t} \nabla \tilde{\mathbf{u}}:\left(\nabla \tilde{\mathbf{u}}+\nabla \tilde{\mathbf{u}}^{T}\right)-\frac{2}{3} \nabla \tilde{\mathbf{u}}\left(3 \mu_{T} \cdot \tilde{\mathbf{u}}+\rho k\right)
$$

where, $\tilde{\mathrm{u}}$ is the mean velocity vector. The empirical constants of the model are $C_{\eta}=0.09 ; C_{\varepsilon 1}=1.44$, $C_{\varepsilon 2}=1.92, \sigma_{\kappa}=1, \sigma_{\varepsilon}=1.3$. The turbulent viscosity can be calculated with the equation $\mu_{t}=\frac{c_{\eta} \rho k^{2}}{\varepsilon}$.

This model of two equations has been used in the description of turbulent jet under supercritical conditions [12].

\section{Methodology}

The objective of this study is to investigate the effects of temperature and pressure in jet velocity of a three-dimensional flow in SAS process and the work of Franceschi [13] was chosen as a reference to validate the model. The experimental apparatus consists of a tubular precipitator of approximately $600 \mathrm{~mL}$ volume. The device design is shown in Figs. 1 and 2 . Table 1 summarizes the experimental conditions investigated. Under these conditions, there is complete miscibility between $\mathrm{CO}_{2}$ and dichloromethane (single phase region).

The simulations were made from mixed mesh with tetrahedral elements and hexahedral elements and a total number of $1.028 \times 10^{6}$ cells. The mesh and geometry details associated with it are shown in Figs. 1 and 2. The details of the inner cell distribution and refining regions at the exit of the capillary and close to 
the exit of the mixing tube can be seen in Fig. 2 which shows a cutting plane at the center of the chamber.

The chamber has an internal diameter of $80 \mathrm{~mm}$ and inside length of $120 \mathrm{~mm}$, capillary length coaxial injection of $98 \mathrm{~mm}$ and the diameter of the inlet of $\mathrm{CO}_{2}$ is $0.97 \mathrm{~mm}$ and the solution inlet diameter is 0.1 $\mathrm{mm}$, and the capillary tube starts at $10 \mathrm{~mm}$ inside the chamber. Contains output by the lid, this being a tube

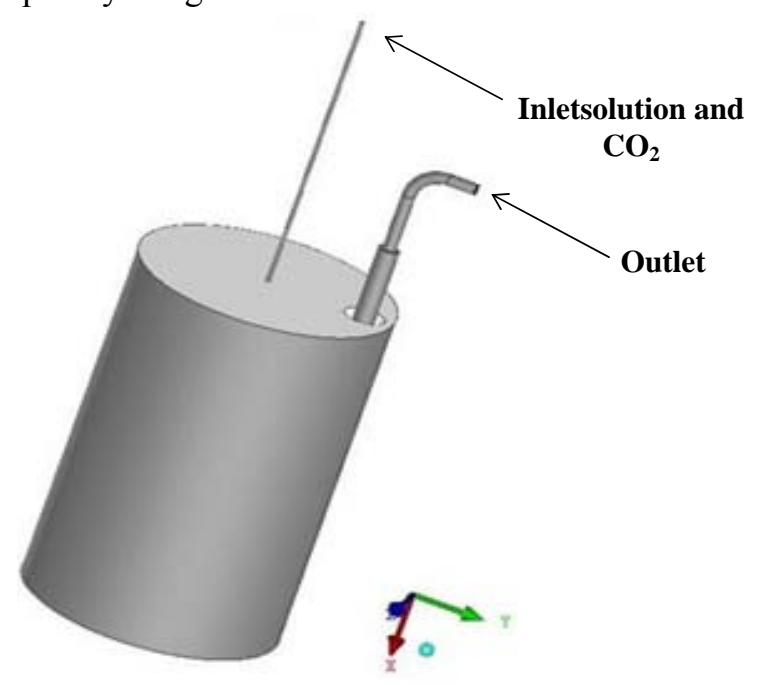

Fig. 1 Mesh with detail geometry with coaxial input and $\mathrm{CO}_{2}$ solution.

Fig. 2 Aspects of the internal elements of the mesh.

\section{Table 1 Operating conditions.}

\begin{tabular}{lll}
\hline Simulation & Temperature $(\mathrm{K})$ & Pressure (bar) \\
\hline 1 & 308.15 & 80 \\
2 & 313.15 & 80 \\
3 & 318.15 & 80 \\
4 & 308.15 & 120 \\
5 & 313.15 & 120 \\
6 & 318.15 & 120 \\
7 & 308.15 & 160 \\
8 & 313.15 & 160 \\
9 & 318.15 & 160 \\
\hline
\end{tabular}

of length $164 \mathrm{~mm}$ and internal diameter $6.34 \mathrm{~mm}$ starting at $35 \mathrm{~mm}$ from the bottom of the chamber and the end of the outlet tube diameter is $3.87 \mathrm{~mm}$.

To solve the system of Eqs. (1)-(8), we used the ANSYS FLUENT 14.5 software, which makes use of the finite volume method. The segregated solver was employed through the SIMPLEC algorithm, whose goal is to solve the linear systems in each equation one by one, updating its coefficients due to nonlinearities and the coupling between variables. 
As convergence criteria was considered the residual sum below $10^{-5}$ for all variables and imposed the closure of mass balance $\left|m_{\mathrm{CO}_{2}}+m_{\text {sol }}-m_{\text {out }}\right|<10^{-11}$.

\section{Results and Discussion}

The results obtained from simulations performed by the software for carbon dioxide mixture, dichloromethane and the grape seed extract, since the presence of the grape seed extract in the system does not change the phase behavior of the binary solvent system plus antisolvent [13] so that still considered the system in a single phase.

\subsection{Effect of Pressure}

Experimental studies have shown that the pressure directly affects the size, size distribution and morphology of the particle, mostly related to temperature, antisolvent flow rate and the concentration of the solute [13].

Analyzing Figs. 3-5, there is the influence on the magnitude of the velocity obtained by the centerline of the precipitation chamber. As the pressure decreases, the jet velocity increases, which results in homogeneous and nearly spherical particles, as demonstrated experimentally by Petit-gas et al. [14] for various mixtures in the SAS process.

Under these operating conditions and according to the results, the most advantageous option would be the use of lower pressure, as experimentally obtained for Franceschi [13] in a precipitation chamber.

\subsection{Effect of Temperature}

With respect to the influence of temperature on the velocity of the jet can be seen in Figs. 6-8 that their maximum values promoted increase of jet velocity and thus increase the yield of precipitated particles, as described by Bashipour and Ghoreishi [15] when extract $\beta$-carotene from the aloe vera plant.

In addition to these operating conditions, other factors may also influence the velocity of the jet and hence the particle size. Cases such as the size of the precipitation chamber, flows and concentrations of the components of the mixing chamber must also be analyzed.

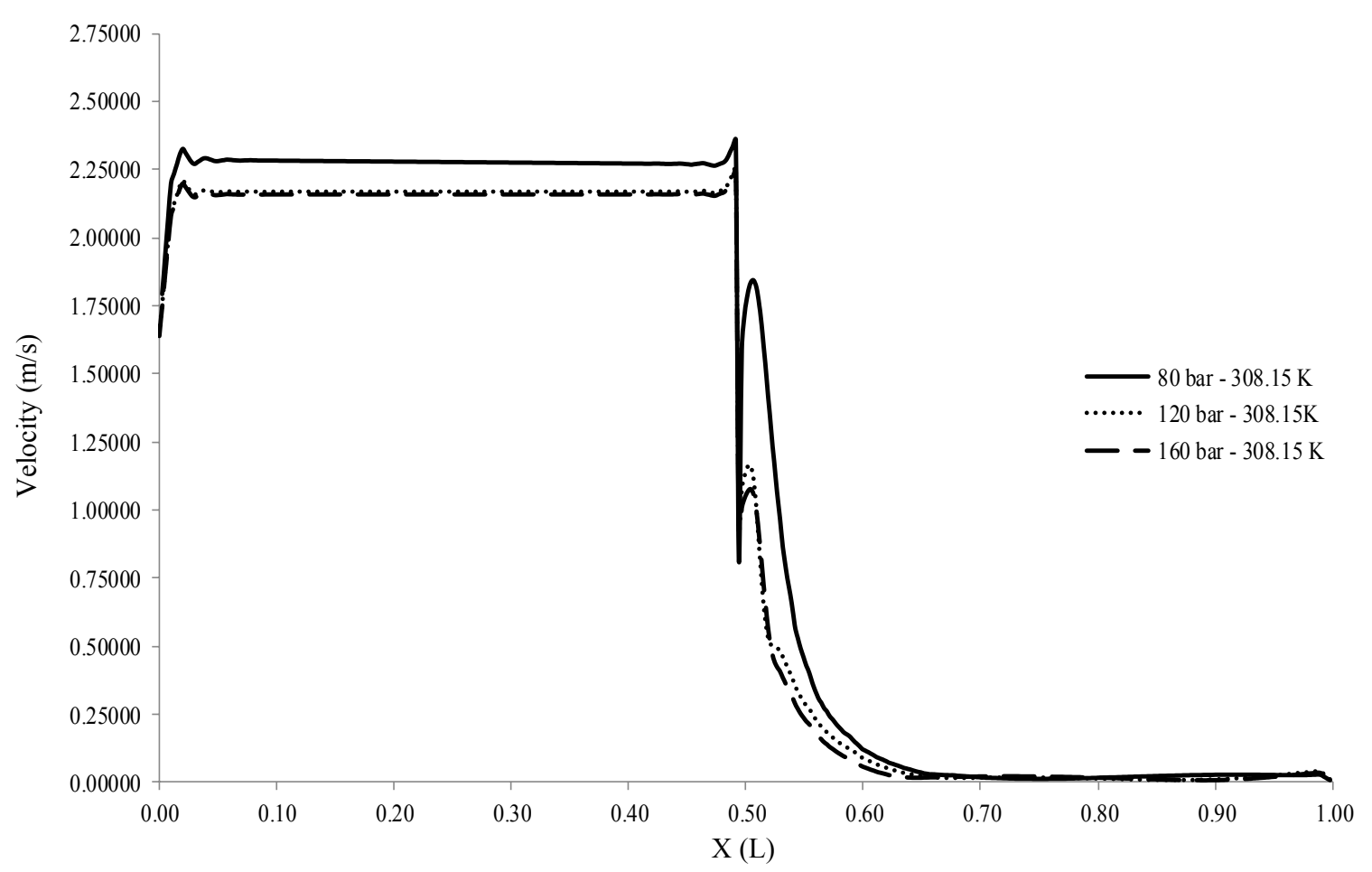

Fig. 3 Values of the magnitude of the velocity along the axis of the chamber with a temperature of $308.15 \mathrm{~K}$. 


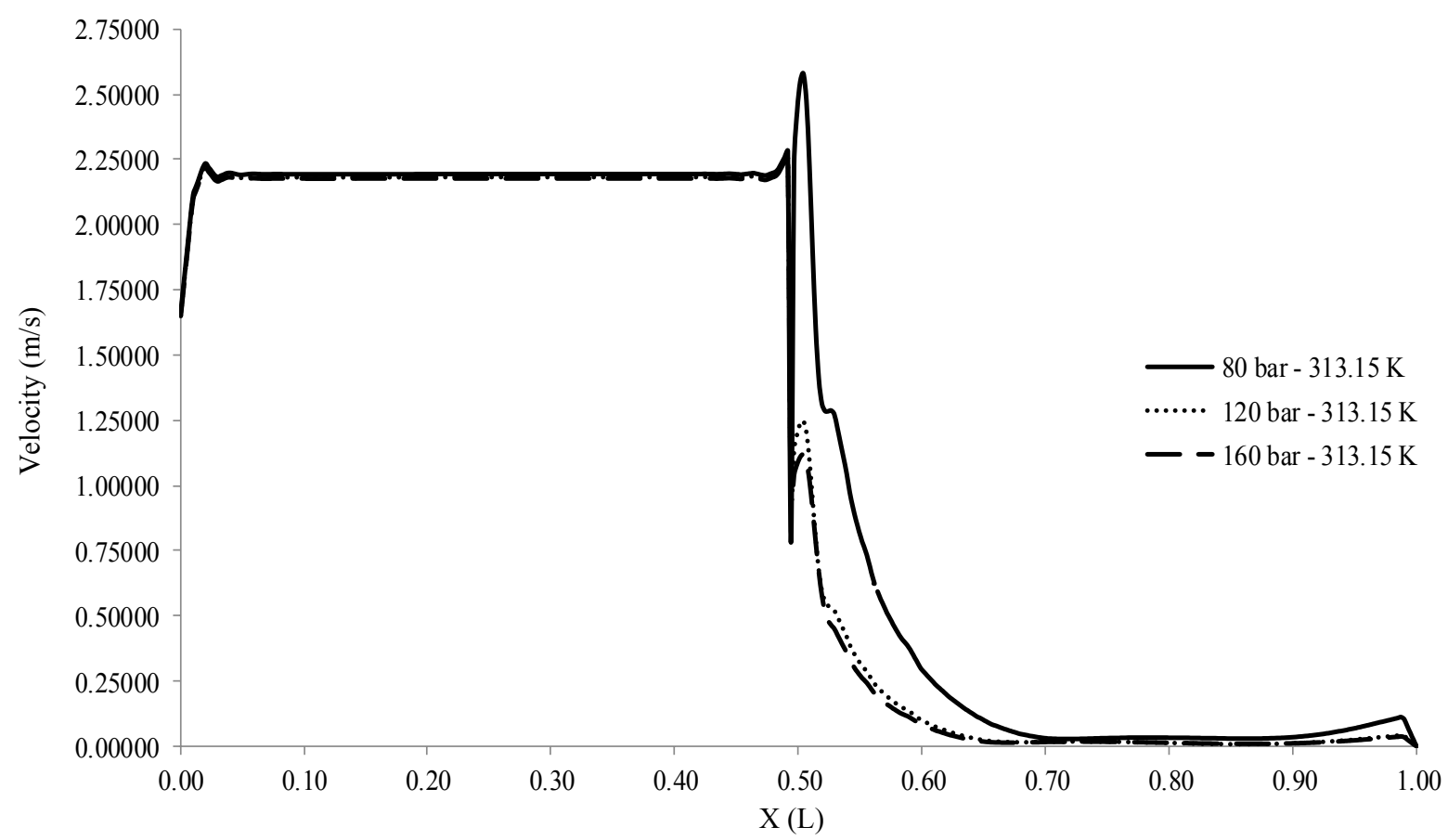

Fig. 4 Values of the magnitude of the velocity along the axis of the chamber with a temperature of $313.15 \mathrm{~K}$.

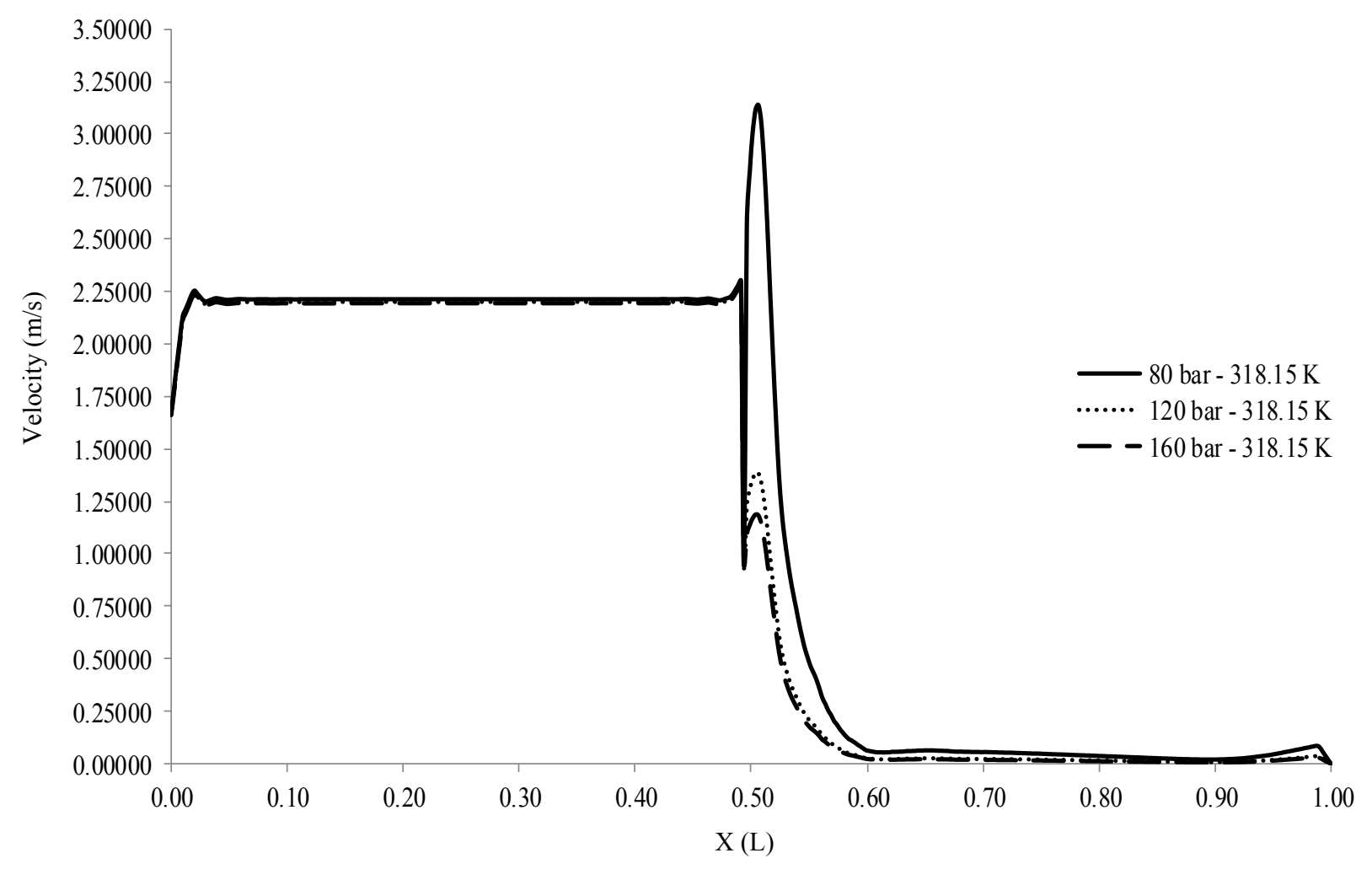

Fig. 5 Values of the magnitude of the velocity along the axis of the chamber with a temperature of $318.15 \mathrm{~K}$. 


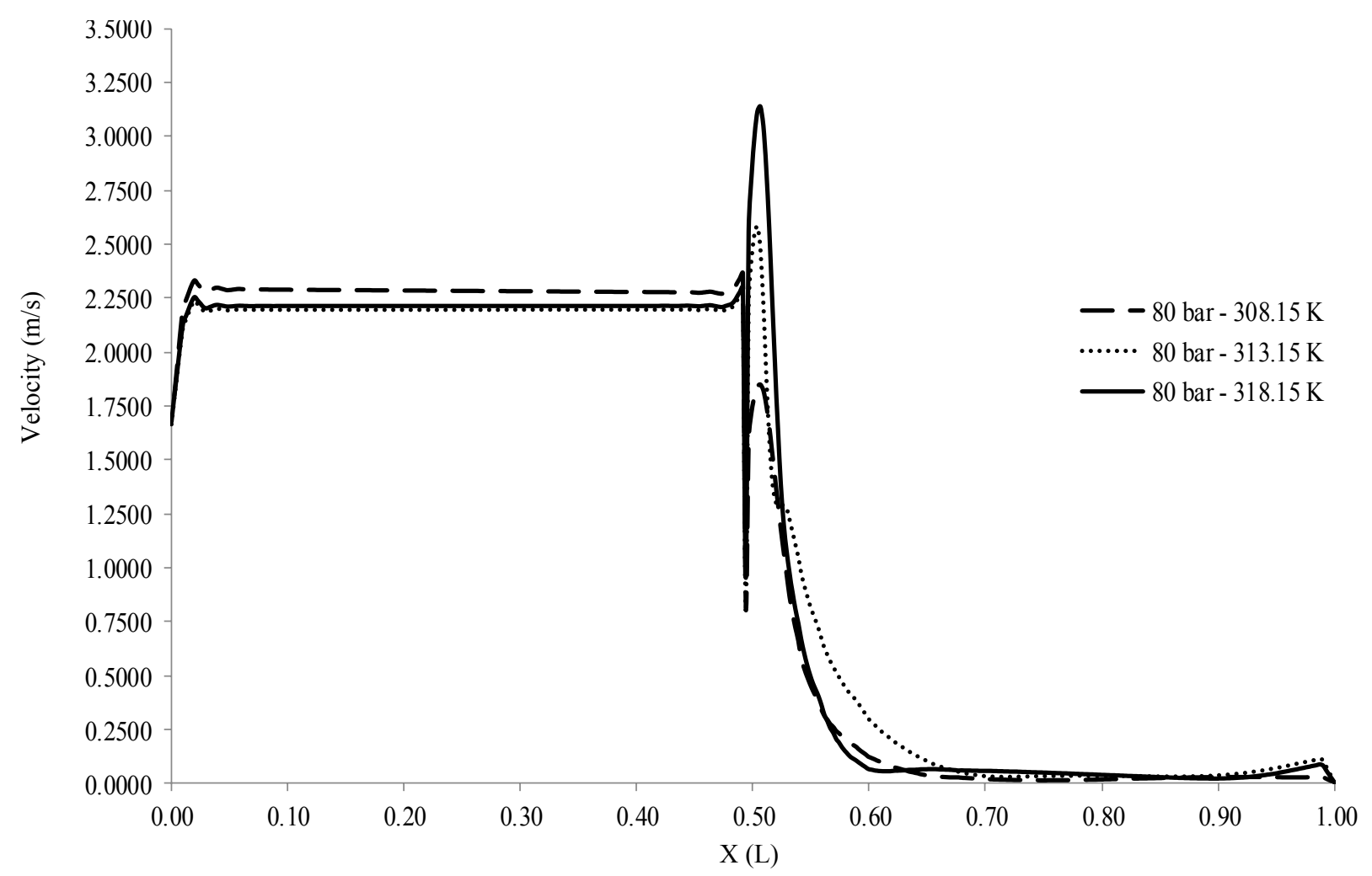

Fig. 6 Values of the magnitude of the velocity along the axis of the chamber with a pressure of $80 \mathrm{bar}$.

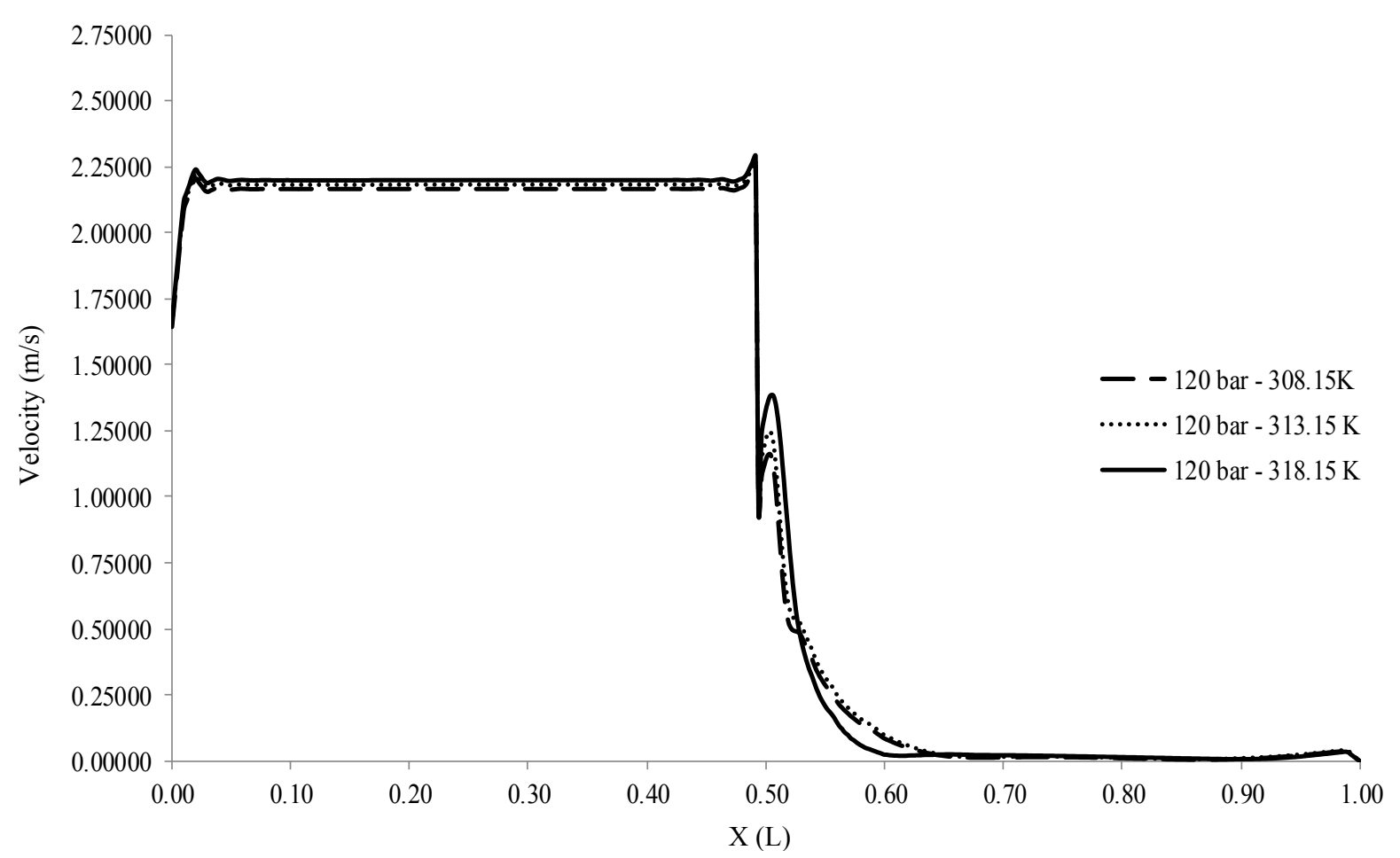

Fig. 7 Values of the magnitude of the velocity along the axis of the chamber with a pressure of 120 bar. 


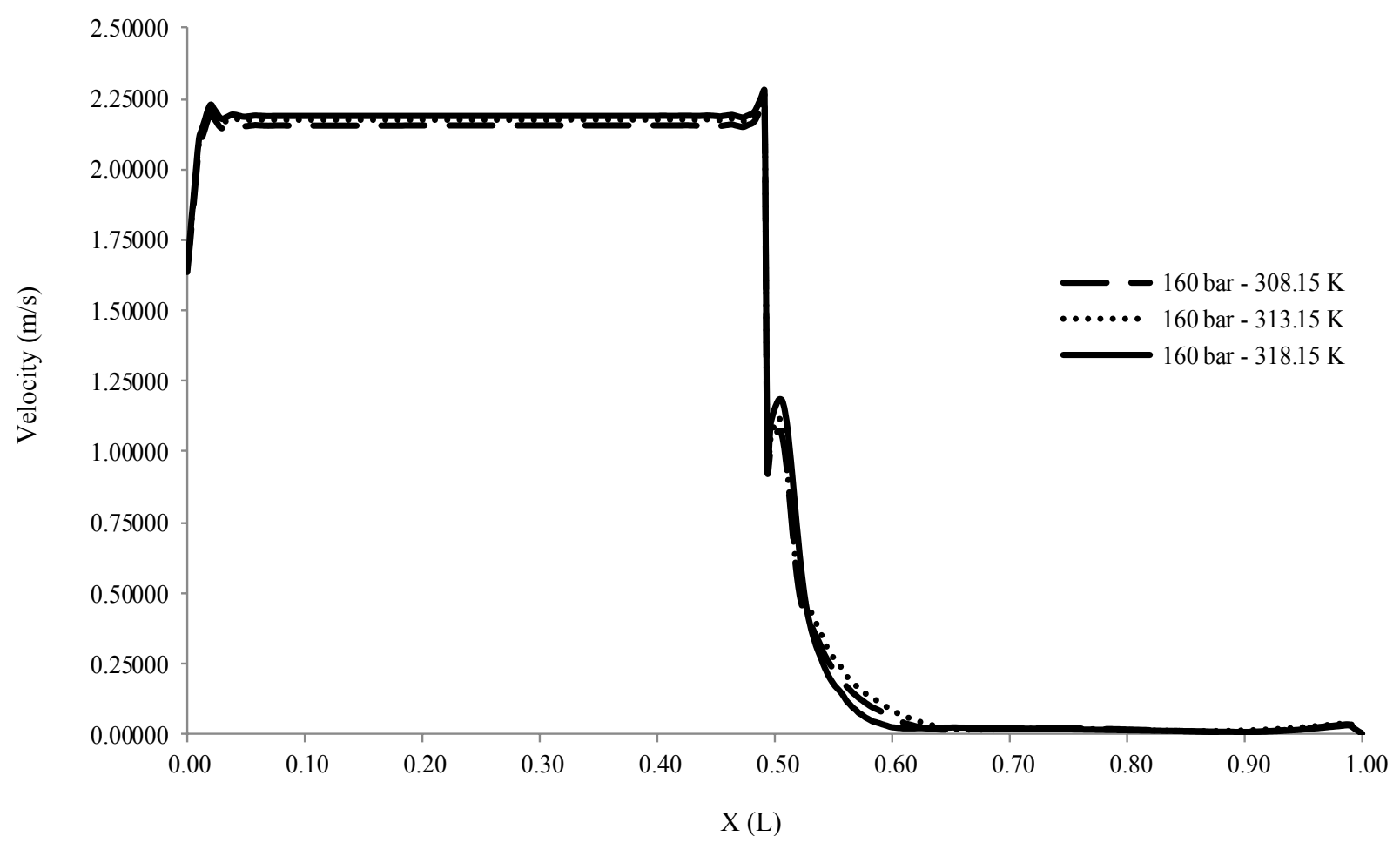

Fig. 8 Values of the magnitude of the velocity along the axis of the chamber with a pressure of $160 \mathrm{bar}$.

\section{Conclusions}

A model used to describe the velocity of the jet in the precipitation of the grape seed extract employing the SAS process was developed. The model includes the density of the mixture via Peng and Robinson [16] equation of state with quadratic mixing rules of Van der Waals for the analysis of the mixture behavior. For the investigated variables, pressure and temperature, results showed they influence the velocity of the jet solution which experimentally impacts the size and morphology of the particles with a stronger effect at lower pressure and higher temperature operation.

The predictions matched well with the experimental data in all cases studied, and although they have not yet combined with a population balance that determines the size and size distribution of particles, the model is able to explain the experimental trend in the jet velocity of a three-dimensional flow and offers a valuable insight into the process. The next steps of this work will be toward another validation of the model in a wide range of operating conditions. In particular, the cases in which the mixture affects precipitation will be investigated. A follow up study will also examine the range of Reynolds numbers for which the effects of turbulence can be optimized, resulting in a final product of good quality.

\section{References}

[1] Jung, J.; Perrut, M. Particle Design Using Supercritical Fluids. Journal of Supercritical Fluids 2001, 20, 179-197.

[2] Reverchon, E.; Adami, R.; Caputo, G.; Marco, I. Spherical Microparticles Production by Supercritical Antisolvent Precipitation. Journal of Supercritical Fluids 2008, 47, 70-84.

[3] Martin, A.; Cocero, M. J. Numerical Modeling of Jet Hydrodynamics, Mass Transfer and Crystallization Kinetics in the Supercritical Antisolvent (SAS) Process. Journal of Supercritical Fluids 2004, 32, 203-219.

[4] Shekunov, B. Y.; Baldyga, J.; York, P. Particle Formation by Mixing with Supercritical Antisolvent at High Reynolds Numbers. Chemical Engineering Science 2001, 56, 2421-2433.

[5] Baldyga, J.; Makowski, L.; Orciuch, W. Interaction between Mixing, Chemical Reactions and Precipitation. Industrial \& Engineering Chemistry Research 2005, 44, 5342-5352.

[6] Werling, J. O.; Debenedetti, P. G. Numerical Modelling of Mass Transfer in the Supercritical Antisolvent Process: Miscible Condition. Journal of Supercritical Fluids 2000, 
18, 11-21.

[7] Chavez, F.; Debenedetti, P.; Luo, J.; Dave, R.; Pfeffer, R. Estimation of the Characteristic Time Scales in the Supercritical Antisolvent Process. Industrial \& Engineering Chemistry Research 2003, 42, 3156-3162.

[8] Pratsinis, S. E. Simultaneous Nucleation, Condensation and Coagulation in Aerosol Reactors. Journal Colloid and Interface Science 1988, 124, 416-429.

[9] Henczka, M.; Baldyga, J.; Shekunov, B. Y. Particle Formation by Turbulent Mixing with Supercritical Antisolvent. Chemical Engineering Science 2005, 60, 2193-2201.

[10] Baldyga, J. Turbulent Mixer Model with Application to Homogeneous, Instantaneous Chemical Reactions. Chemical Engineering Science 1989, 44, 1175-1182.

[11] Oschwald, M.; Smith, J. J.; Branam, R.; Hussong, J.; Schik, A.; Chehroudi, B.; et al. Injection of Fluids into Supercritical Environments. Combustion Science Technology 2006, 178, 49-100.
[12] Sierra-pallares, J.; Marchisio, D. L.; Parra-santos, M.; Garcia-serna, J.; Castro, F.; Cocero, M. A Computational Fluid Dynamics Study os Supercritical Antisolvent Precipitation: Mixing Effects on Particle Size. AIch. E. Journal 2012, 58, 385-398.

[13] Franceschi, E. Precipitation and Encapsulation of $\beta$-carotene in PHBV Using Supercritical Technology. Ph.D. Thesis, Federal University of Santa Catarina, 2009.

[14] Petit-gas, T.; Boutin, O.; Raspo, I.; Badens, E. Role of Hydrodynamics in Supercritical Antisolvent Processes. Journal of Supercritical Fluids 2009, 51, 248-244.

[15] Bashipour, F.; Ghoreishi, S. M. Experimental Optimization of Supercritical Extraction of $\beta$-carotene from Aloe Bar Badensis Miller via Genetic Algorithm. Journal of Supercritical Fluids 2012, 72, 312-319.

[16] Peng, D. Y.; Robinson, D. B. A New Two-Constant Equation of State. Industrial Engineering Chemistry Fundamentals 1976, 15, 59-72. 